BARROS, I.O. et al. Eficácia anti-helmíntica da ivermectina $1 \%$ sobre nematódeos gastrintestinais de caprinos em Mossoró, Rio Grande do Norte, Brasil. PUBVET, Londrina, V. 8, N. 2, Ed. 251, Art. 1663, Janeiro, 2014.

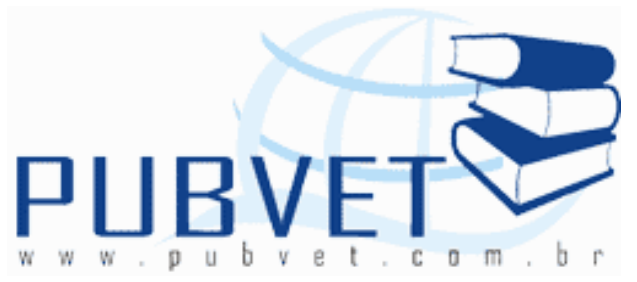

PUBVET, Publicações em Medicina Veterinária e Zootecnia.

\title{
Eficácia anti-helmíntica da ivermectina 1\% sobre nematódeos gastrintestinais de caprinos em Mossoró, Rio Grande do Norte, Brasil
}

Isabella de Oliveira Barros ${ }^{1}$, Ivana Cristina Nunes Gadelha ${ }^{1}$, Isabel Bezerra Ribeiro Albuquerque ${ }^{2}$, Muriel Magda Lustosa Pimentel ${ }^{2}$, Zuliete Aliona Araújo de Souza Fonseca ${ }^{1}$, Wesley Adson Costa Coelho ${ }^{1}$, Josivania Soares Pereira ${ }^{1 *}$, Sílvia Maria Mendes Ahid ${ }^{3}$.

1 Doutorandos do Programa de Pós-graduação em Ciência Animal da Universidade Federal Rural do Semi-Árido (UFERSA).

${ }^{2}$ Mestrandas do Programa de Pós-Graduação em Ciência Animal da UFERSA.

*e-mail para correspondência: josigej@ufersa.edu.br

${ }^{3}$ Docente da UFERSA.

\section{Resumo}

É relevante estudar a resistência anti-helmíntica na caprinocultura, já que a produção e a produtividade deste segmento é prejudicada devido ainfecção por endoparasitas. Desta forma, objetivou-se avaliar a ação anti-helmíntica da ivermectina $1 \%$ sobre nematódeos gastrintestinais de caprinos naturalmente infectados, oriundos do Setor deovinocaprinocultura da Universidade Federal Rural do Semi-Árido em Mossoró, RN. Foram utilizados 20 caprinos SRD, divididos igualmente em grupo controle e grupo tratado, sendo neste último administrado, ivermectina $1 \%$ no primeiro dia de experimento. As coletas de fezes foram feitasantes da administração do fármaco e após dez dias do 
BARROS, I.O. et al. Eficácia anti-helmíntica da ivermectina $1 \%$ sobre nematódeos gastrintestinais de caprinos em Mossoró, Rio Grande do Norte, Brasil. PUBVET, Londrina, V. 8, N. 2, Ed. 251, Art. 1663, Janeiro, 2014.

experimento. As amostras foram submetidas a exames coproparasitológicos no Laboratório de Parasitologia Animal da UFERSA, onde a análise da eficiência da droga foi feita pelo teste de redução na contagem de ovos nas fezes e a diferenciação das larvas infectantes pela coprocultura, sendo os dados analisados estatisticamente pelo programa RESO 2.0. A eficiência da ivermectina foi de $80 \%$, com intervalo de confiança e limite superior de $92 \%$. Sendo os helmintos mais resistentes à ação da ivermectina, o gênero Haemonchus, com redução de $77 \%$ da carga parasitária.

Palavras-chave: Caprinocultura, OPG, Helmintos.

\title{
Anthelmintic efficacy of ivermectin $1 \%$ of gastrointestinal nematodes of goats in Mossoró, Rio Grande do Norte, Brasil
}

\begin{abstract}
It is relevant to study the anthelmintic resistance in goat, since the production and productivity of this segment is impaired due to infection by endoparasites. Thus, this study aimed to evaluate the anthelmintic activity of ivermectin $1 \%$ of gastrointestinal nematodes of naturally infected goats, sheep and goat farming sector coming from the Universidade Federal rural do Semi-Árido in Mossoró, RN. SRD 20 goats were used, divided into control and experimental group, with the latteradministeredivermectin $1 \%$ on the first day of the experiment. The fecal samples were taken before drug administration and after ten days of the experiment. The samples were subjected to fecal examination in the Parasitology Laboratory Animal UFERSA where analysis of the efficiency of the drug test was reduction in faecal egg countand differentiation of infective larvae by fecal culture, and the data were statistically analyzed by the RESO 2.0. The efficacy of ivermectin was $80 \%$, with a confidence intervalandupper limitof $92 \%$. Helminths being more resistant to the action of ivermectin, the genus Haemonchus, with $77 \%$ reduction of parasite load.
\end{abstract}

Keywords: Goat, OPG, Helminths. 
BARROS, I.O. et al. Eficácia anti-helmíntica da ivermectina $1 \%$ sobre nematódeos gastrintestinais de caprinos em Mossoró, Rio Grande do Norte, Brasil. PUBVET, Londrina, V. 8, N. 2, Ed. 251, Art. 1663, Janeiro, 2014.

\section{INTRODUÇÃO}

A caprinocultura é uma atividade muito explorada em países tropicais, principalmente no Brasil, pois apresenta grande importância socioeconômica, visandoàprodução de carne, leite e pele (VIEIRA, 2003). O rebanho caprino brasileiro apresenta 9,3 milhões de cabeças em seu contingente, estando a maior concentração na região Nordeste, onde são criados principalmente sob sistemas de produção poucos tecnificados, utilizando animais com baixo potencial genético para atender as exigências do mercado consumidor em termos de regularidade e qualidade (IBGE, 2011).

Na região semiárida, o desenvolvimento da criação caprina é bastante evidente, porém o parasitismo por nematódeos tem sido um dos principais problemas sanitários. Apesar do progresso geral, o parasitismo por nematódeos gastrintestinais continuam a ser uma das principais causas de doenças subclínicas, queda na produção e perda econômica para o setor (MOLENTO et al., 2011; VERÍSSIMO et al., 2012 ).

Dentre os nematódeos que parasitam pequenos ruminantes 0 Haemonchus contortus apresenta a maior preocupação em regiões tropicais e subtropicais, em decorrência da sua prolificidade e grande potencial patogênico (NEMATOLLAHI et al., 2007). Os sinais clínicos observado pelo parasitismo por H. contortus podem ser diarreia, desidratação, edema, hidrotórax, hidroperitônio, taxas de crescimento reduzidaspodendo levar animais a óbito (ZAJAC, 2006), sendo a anemia é o principal achado laboratorial de infecções pelo referido nematódeo (OSMAN et al., 2004; AMEEN et al., 2006; OKAIYETO et al., 2010). Além deste, outros helmintos gastrintestinais tais como os Strongyloides sp., Oesophagostomum sp. e Trichostrongylus sp. são observados em associação ao $H$. contortus parasitando pequenos ruminantes do Rio Grande do Norte (AHID et al., 2008).

O controle das endoparasitoses é essencial para o sucesso da produção de pequenos ruminantes. Desta forma, existem vários princípios ativos que estão sendo utilizados no tratamento contra nematódeos, dentre eles 
BARROS, I.O. et al. Eficácia anti-helmíntica da ivermectina $1 \%$ sobre nematódeos gastrintestinais de caprinos em Mossoró, Rio Grande do Norte, Brasil. PUBVET, Londrina, V. 8, N. 2, Ed. 251, Art. 1663, Janeiro, 2014.

destacam-se os grupos dos benzimidazóis (Albendazole, fenbendazole e oxfendazole); das avermectinas (Ivermectina); dos imidazotiazoles (Cloridrato de levamisole) e dos salicilanilideos (Closantel sódico) (BORGES, 2003). O uso frequente dessas bases podem acarretar a resistência.

Segundo Conder e Campbell (1995), resistência parasitária é o fenômeno pelo qual a droga não consegue manter a mesma eficácia contra parasitas, quando utilizada nas mesmas condições, após determinado período de tempo. O diagnóstico é positivo para "resistência" quando uma determinada droga que apresentava redução da carga parasitária acima de 95\% decresce a nível inferior a este valor contra o mesmo organismo depois de determinado período (MOLENTO, 2005).

A eficácia das drogas diminui consideravelmente devido ao caráter seletivo, favorecendo a permanência de organismos resistentes e a eliminação de indivíduos susceptíveis, tornando o aparecimento da resistência praticamente inevitável transferindo as características genéticas para próximas gerações (COELHO, 2010), em que a população de helmintos resistente não é alterada com o tratamento anti-helmíntico, ocorrendo então, uma mudança da característica genética da populaçãoparasitária (MOLENTO, 2005).

Nesta linha, este trabalho teve por objetivo avaliar a eficácia da ivermectina $1 \%$ sobre nematódeos gastrintestinais de caprinos naturalmente infectados localizados no setor de caprinocultura da Universidade Federal Rural do Semi-Árido (UFERSA), no município de Mossoró, RN.

\section{MATERIAL E MÉTODOS}

O experimento ocorreu no mês de abril 2013. Foram utilizados 20 caprinos SRD, escolhidos aleatoriamente com vermifugação realizada a mais de 45 dias, mantidos em regime intensivo, divididos em dois grupos, sendo 10 animais para o grupo controle (grupo I) e 10 para tratado (grupo II). Todos os caprinos foram provenientes do Setor de ovinocaprinocultura, da Universidade Federal Rural do Semi-Árido, Mossoró-RN. 
BARROS, I.O. et al. Eficácia anti-helmíntica da ivermectina $1 \%$ sobre nematódeos gastrintestinais de caprinos em Mossoró, Rio Grande do Norte, Brasil. PUBVET, Londrina, V. 8, N. 2, Ed. 251, Art. 1663, Janeiro, 2014.

Os animais foram identificados com um colar numerado de coloração diferente para cada grupo. Onde o grupo I não recebeu nenhum tratamento, e o grupo II, recebeu no primeiro dia de experimento, ivermectina ${ }^{\circledR} 1 \%$ em dose recomendada pelo fabricante.

Foram realizadas coletas de fezes individuais, diretamente da ampola retal, nos caprinos infectados naturalmente por nematódeos gastrintestinais, antes e 10 dias pós tratamento. Estas foram identificadas e encaminhadas para processamento e análise no Laboratório de Parasitologia Animal da UFERSA.

Cada amostra foi processada individualmente para realização da contagem de ovos por grama de fezes (OPG), pela técnica de Gordon e Whitlock (1939) e coprocultura segundo técnica de Ueno e Gonçalves (1998).

Os dados foram analisados estatisticamente pelo programa RESO 2.0 (WURSTHORN; MARTIN, 1990), em que a resistência está presente se a percentagem de redução da contagem de ovos for inferior a $95 \%$ e se o limite inferior do intervalo de confiança a $95 \%$ for menor que $90 \%$.

\section{RESULTADOS E DISCUSSÃO}

No presente estudo ficou claramente evidente a resistência à ivermectina pelos endoparasitos encontrados nas fezes dos caprinos avaliados, apresentando OPG médio de 1.570 e 310 ovos por grama de fezes no grupo controle e tratado com ivermectina ${ }^{\circledR} 1 \%$, respectivamente, onde a eficácia da droga apresentou média de redução de $80 \%$, sendo que os valores variaram de 49 a 92\%, o que demonstra resistência ao principio no rebanho da UFERSA. Esses dados corroboram com Molento (2004), que descreve um diagnóstico positivo para resistência, quando uma determinada droga que apresentava redução acima de $99 \%$ da carga parasitária apresenta redução menor que 95\% contra determinado organismo após determinado período de tempo.

A baixa eficácia da ivermectina $1 \%$ encontrada no presente estudo, corroboram com dados descritos por Melo et al.(2003) e Coelho et al. (2010) 
BARROS, I.O. et al. Eficácia anti-helmíntica da ivermectina $1 \%$ sobre nematódeos gastrintestinais de caprinos em Mossoró, Rio Grande do Norte, Brasil. PUBVET, Londrina, V. 8, N. 2, Ed. 251, Art. 1663, Janeiro, 2014.

que descrevem redução variando de 60 a $100 \%$ e 43 a $100 \%$ em caprinos no estado do Ceará (Pentecostes) e Rio Grande do Norte (Mossoró) respectivamente. Já Sousa et al. (2013) observaram alta redução da infecção parasitária na utilização desse fármaco em caprinos leiteiros do sertão da Paraíba (Sumé), onde conseguiu eficácia de 97\% e 97,8\%, após 7 e 14 dias de sua administração, possivelmente em decorrência da não utilização da ivermectina $1 \%$ anteriormente. A ivermectina pertence ao grupo das lactonas macrocíclicas e há muito tempo vem sendo utilizada no controle de ecto e endoparasitas de pequenos ruminantes. Seu uso indiscriminado e sem respeitar o intervalo de aplicação e dose recomendada pelo fabricante tem favorecido o crescimento de casos de resistência.

O gênero de maior prevalência foi Strongyloides (48\%), seguido por Haemonchus (34\%), Trichostrongylus (10\%) e Oesophagostomum (8\%), sendo o gênero Haemonchus o de maior resistência, com redução de OPG variando de 40 a 91\%. Corroborando com Ahid et al. (2008), que descreve como principais nematódeos gastrointestinais de caprinos o Strongyloides sp., seguidos por Haemonchus sp., Trichostrongylus sp. e, em menor escala Oesophagostomum sp., não sendo identificado Ostertagia sp. no município de Mossoró, RN. Sabe-se que parasitismo por esses nematódeos gastrintestinais tem-se constituído em um dos principais fatores limitantes à exploração da caprinocultura no semiárido nordestino, pois provocam nos animais diversos prejuízos como crescimento retardado, perda de peso, redução no consumo de alimentos, queda na produção de leite, baixa fertilidade e, nos casos de infecções maciças, altas taxas de mortalidade (VIEIRA et al., 1991).

Segundo Coelho et al. (2010) a resistência à ivermectina encontra-se bem evidente e distribuída nos caprinos pertencentes a Mossoró-RN, mas em menor proporção ao compará-la com albendazole, tendo em vista que os produtores desta área relataram utilizarem no controle das verminoses com maior frequência os benzimidazóis e lactonas macrocíclicas.

Novos métodos alternativos de controle a verminoses em caprinos devem ser adotados, tais como o método FAMACHA, controle baseado em 
BARROS, I.O. et al. Eficácia anti-helmíntica da ivermectina $1 \%$ sobre nematódeos gastrintestinais de caprinos em Mossoró, Rio Grande do Norte, Brasil. PUBVET, Londrina, V. 8, N. 2, Ed. 251, Art. 1663, Janeiro, 2014.

OPG, seleção genética de animais resistentes, no intuito de minimizar o uso de fármacos e com isso reduzir a resistência parasitária.

\section{CONCLUSÃO}

O presente estudo observou resistência parasitária à ivermectina $1 \%$, sendo recomendado a realização de testes com outros princípios ativos, e adoção de métodos alternativos para controle de endoparasitas em caprinos.

\section{REFERÊNCIAS}

Ahid, S. M. M.; Suassuna, A. C. D.; Maia. M. B.; Costa, V. M. M.; Soares, H.S. 2008. Parasitos gastrintestinais em caprinos e ovinos da região oeste do Rio Grande do Norte, Brasil.Ciência Animal Brasileira, 9 (1): 212-218.

Ameen, S. A.; Joshua, R. A.; Adedeji, O. S.; Ige, A. O.;Oyebanji, B. O.; Ogundola,A. F.;Rafiu, T. A. 2006. Experimental studies on gastro-intestinal nematode infection: Clinical observations and haematological changes followingHaemonchus contortusinfection in West African Dwarf (WAD) kids. Journal of Animal and Veterinary Advances, 5 (6): 511-514.

Borges,C. C. L. 2003. Atividade in vitro de anti-helmínticos sobre larvas infectantes de nematódeos gastrintestinais de caprinos, utilizando a técnica de coprocultura quantitativa (Ueno, 1995).ParasitolLatinoam, 58: 142 - 147.

Coelho, W. A. C.; Ahid, S. M. M; Vieira, L. S; Fonseca, Z. A. A; Silva, I. P. 2010. Resistência anti-helmíntica em caprinos no município de Mossoró, RN.Revista Ciência Animal Brasileira,11 (3): 589-599.

Gordon, H.M.; Whitlock, H.V.A., New technique for counting nematodes eggs in sheep faeces.Journal. Coun. Sci. Ind. Res. Aust.,v.12, p.50-52, 1939.

IBGE - Instituto Brasileiro de Geografia e Estatístico. 2011.Disponível em: http://www.ibge.gov.br. Acessado em 08 de junho de 2013.

Melo, A. C. F. L.; Reis, I. F.; Bevilaqua, C. M. L.; Vieira, L. S.; Echevarria, F. A. M.; Melo, L. M. 2003. Nematodeos resistentes a anti-helmínticos em rebanhos de ovinos e caprinos do estado do Ceará, Brasil. Ciência Rural, 33: 339-344.

Molento M.B., Fortes F., PondelekD., Borges F.A., Chagas A.C.S., Torres Acosta J.F.J., Geldhof P. 2011. Challenges of nematode control in ruminants: Focus on Latin America.

VeterinaryParasitology. 180:126-132.

. 2004. Resistência de helmintos em ovinos e caprinos. Revista Brasileira de Parasitologia Veterinária, 13: 82-85. 
Molento, M. B. 2005. Resistência parasitária em helmintos de equídeos e propostas de manejo. Ciência Rural, 35 (6): 1469-1477.

Nematollahi, A. A.; Eslami.; S. H. Hosseini. 2007. Experimental study on the causative agents of hypobios is of Haemonchus contortusin sheep in Iran. Journal of Animal and Veterinary Advances, 6 (3): 458-460.

Okaiyeto, S. O.; Ajanusi, O. J.; Sackey, A. K.; Tekdek, L. B.. 2010. Changes in some haematological values associated with mixed. Trypanosomacongolenseand Haemonchuscontortusinfection in Yankassa sheep.Veterinary Research, 3: 9-13.

Osman, A.Y.; Abakar, A. D.; Alamin, A. E. 2004. Haematological and biochemical changes following concurrent infections with CoccidiaandHaemonchus contortusin desert lambs. Journal of Animal and Veterinary Advances, 3: 643-647.

Sousa, A. L. S. O.; Athayde, A. C. R.; Olinto, F. A. 2013.Sensibilidade dos nematódeos gastrointestinais de caprinos leiteiros à anti-helmínticos no município de Sumé, Paraíba, Brasil. Agropecuária Científica do Semi-Árido, 9 (2): 33-36.

Ueno, H.; Gonçalves, P.P.C. 1998. Manual para diagnóstico das helmintoses de ruminantes. Salvador: JapanInternationalCooperationAgency, 1998. 143 p.

Veríssimo; C. J.;Niciura, S. C.; Alberto, A. L.; Rodrigues, C. F.; Barbosa, C. M.; Chiebao, D. P.; Cardoso, D.; Da Silva, G. S.; Pereira, J. R.; Margatho, L. F.; Da Costa, R. L.; Nardon, R. F.; Ueno, T. E.;Curci, V. C.; Molento, M. B. 2012. Multidrug and multispecies resistence in sheep flocks from São Paulo state. BrazilianJournalofVeterinaryParasitology. 187 (1-2), 209-216.

Vieira, L. S. 2003. Alternativas de controle da verminose gastrintestinal dos pequenos ruminantes. Circular Técnica, n.29. Sobral: Embrapa CNPC. 10p.

Vieira, L.S.; Cavalcante, A. C. R.; Ximenes, L. J. F. Epidemiologia e Controle das Principais Endoparasitoses de Caprinos e Ovinos. 1991.In: REUNIÃO ANUAL DA SOCIEDADE BRASILEIRA DE ZOOTECNIA, 28. 1991. João Pessoa: Sociedade Brasileira de Zootecnia. Caprinocultura e Ovinocultura, p. 27-36,

Wursthorn, L.; Martin, P. 1990.Reso: faecal egg count reduction test (FECRT) Analysis Program. 2.01. Parkville: CSIRO Animal Health Research Laboratory.

Zajac, A. M., 2006. Gastrointestinal nematodes of small ruminants: Life cycles, anthelmintics and diagnosis. Veterinary Clinics Food and Animal Practics, 22: 529-541. 